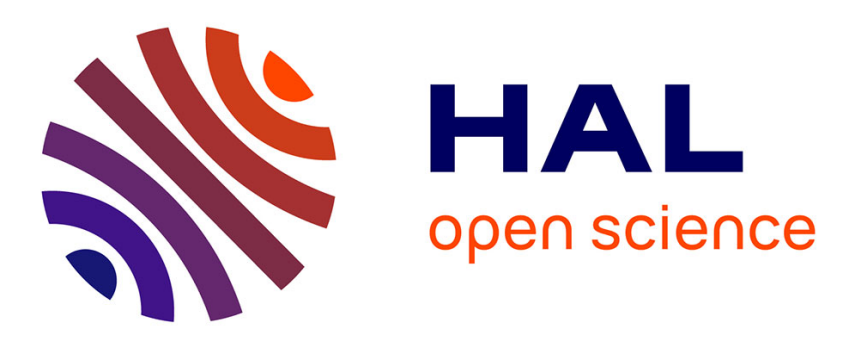

\title{
Discriminating real objects in radar imaging by exploiting the squared modulus of the continuous wavelet transform
}

M. Tria, Jean-Philippe Ovarlez, Luc Vignaud, Juan Carlos Castelli, Messaoud Benidir

\section{To cite this version:}

M. Tria, Jean-Philippe Ovarlez, Luc Vignaud, Juan Carlos Castelli, Messaoud Benidir. Discriminating real objects in radar imaging by exploiting the squared modulus of the continuous wavelet transform. IET Radar Sonar and Navigation, 2007, 1 (1), pp.27. 10.1049/iet-rsn:20050124 · hal-03149811

\section{HAL Id: hal-03149811 https://hal.science/hal-03149811}

Submitted on 10 Feb 2022

HAL is a multi-disciplinary open access archive for the deposit and dissemination of scientific research documents, whether they are published or not. The documents may come from teaching and research institutions in France or abroad, or from public or private research centers.
L'archive ouverte pluridisciplinaire HAL, est destinée au dépôt et à la diffusion de documents scientifiques de niveau recherche, publiés ou non, émanant des établissements d'enseignement et de recherche français ou étrangers, des laboratoires publics ou privés. 


\title{
Discriminating real objects in radar imaging by exploiting the squared modulus of the continuous wavelet transform
}

\author{
M. Tria, J.P. Ovarlez, L. Vignaud, J.C. Castelli and M. Benidir
}

\begin{abstract}
New technique based on continuous wavelet transform (CWT) for classifying objects in synthetic aperture radar (SAR) imaging is presented. The CWT allows to analyse two-dimensional SAR images to highlight the frequency and angular behaviour of the scatterers ref. 10, 11. This technique allows to build a SAR hyperimage, that is, a four-dimensional data cube which represents for each spatial location $(x, y)$ of the scatterer in the image, its frequency and angular energy behaviour. When analysing different targets, objects or areas in SAR images, it has been recently observed that some scatterers belonging to a same class of objects could have similar frequency and angular energy responses. The previous observations have motivated the determination to exploit these energy responses to discriminate these objects. This discrimination is performed by frequency and angular correlations between the response of a particular scatterer (measured) and those of all the scatterers in the SAR image. Some examples of discrimination from real SAR data are presented and show an interest of the method for target classification and recognition for SAR imaging.
\end{abstract}

\section{Introduction}

The synthetic aperture radar (SAR) imaging process $[1,2]$ consists in the formation of high-resolution images. To do so, a moving radar emits pulses and collects the signals reflected by scatterers (see Fig. 1). The imaging plane is labelled using an $x-y$ Cartesian coordinate system with origin at a reference point $\mathcal{O}$. We assume that the radar moves in a straight line and in a direction parallel to the cross-range direction. The radar position is described by azimuth angle $\theta$ defined counterclockwise from the $y$-direction. Moreover, we suppose far field conditions, and therefore we obtain plane-wave incidence on objects. In addition, we assume high-frequency radar measurements and consequently the scattering response of a man-made target is well approximated as a sum of responses from individual scatterers. In traditional SAR imaging, we consider a target as a collection of ideal scatterers, each with a complex amplitude $\sigma_{i}$ and position $\boldsymbol{r}_{i}$. Hence, the scattering model for a given polarisation is [3-5]

$$
H(\boldsymbol{k})=\sum_{i=1}^{N} \sigma_{i} \exp \left(-j 2 \pi \boldsymbol{k} \cdot \boldsymbol{r}_{i}\right)
$$

where $\boldsymbol{k}$ is the wavenumber vector in the direction of the scattered field. An example of monostatic SAR system is illustrated in Fig. 1 where the radar is carried

\footnotetext{
doi:10.1049/iet-rsn:20050124

Paper first received 15th October 2005 and in revised form 24th May 2006 M.

Tria is with the LIS-ENSIEG, BP 46, Saint Martin d'Hères 38402, France

J.P. Ovarlez, L. Vignaud and J.C. Castelli are with the ONERA, DEMR, BP 72, Chatillon 92322, France

M. Benidir is the with the L.S.S. (UMR 8506), Supelec, Gif-sur-Yvette Cedex 91192, France

E-mail: mohamed_tria@hotmail.com
}

by a moving aircraft. The position vector is $\boldsymbol{r}=(x, y)^{\mathrm{T}}$ where $x$ and $y$ represent, respectively, the slant-range and cross-range locations and the wavenumber vector $\boldsymbol{k}$ is

$$
\boldsymbol{k}=\left(\begin{array}{l}
k_{x} \\
k_{y}
\end{array}\right)=\left(\begin{array}{l}
k \cos \theta \\
k \sin \theta
\end{array}\right)
$$

where $k=2 f / c$ is the wave number with $f$ the frequency and $c$ the speed of light, $\theta$ corresponds to the observation aspect.

Considering the scattering model in (1), the complexvalued image $I(\boldsymbol{r})$ is obtained by applying the 2DFourier transform on $H(\boldsymbol{k})$

$$
\begin{aligned}
I(\boldsymbol{r}) & =\underset{\boldsymbol{k} \rightarrow \boldsymbol{r}}{\mathcal{F}^{-1}} H(\boldsymbol{k}) \\
& =\sum_{i=1}^{N} \sigma_{i} \delta\left(\boldsymbol{r}-\boldsymbol{r}_{i}\right)
\end{aligned}
$$

However, the model proposed in (1) is less physically accurate for high relative bandwidth signals or when the SAR data are collected over a broad angular aperture. Indeed, if the radar measurements are carried out over a broad bandwidth and wide angular window, we have to suggest a model that takes the scattering phenomenology into account. This model is expressed as a summation of point scatterers multiplied by their respective frequency- and aspect-dependent amplitude function

$$
H(\boldsymbol{k})=\sum_{i=1}^{N} \sigma_{i}(f, \theta) \exp \left(-j 2 \pi \boldsymbol{k} \cdot \boldsymbol{r}_{i}\right)
$$

where $\sigma_{i}(f, \theta)$ is a 2D-function determined by the geometry, composition and orientation of the scattering mechanism. First, the geometrical theory of diffraction (GTD) $[6,7]$ predicts that the frequency-dependent amplitude is of the form $(j f)^{\alpha}$ where $\alpha$ is related to the scatterer shape. For example, the scatterer can be a corner or a flat plate. Second, the 


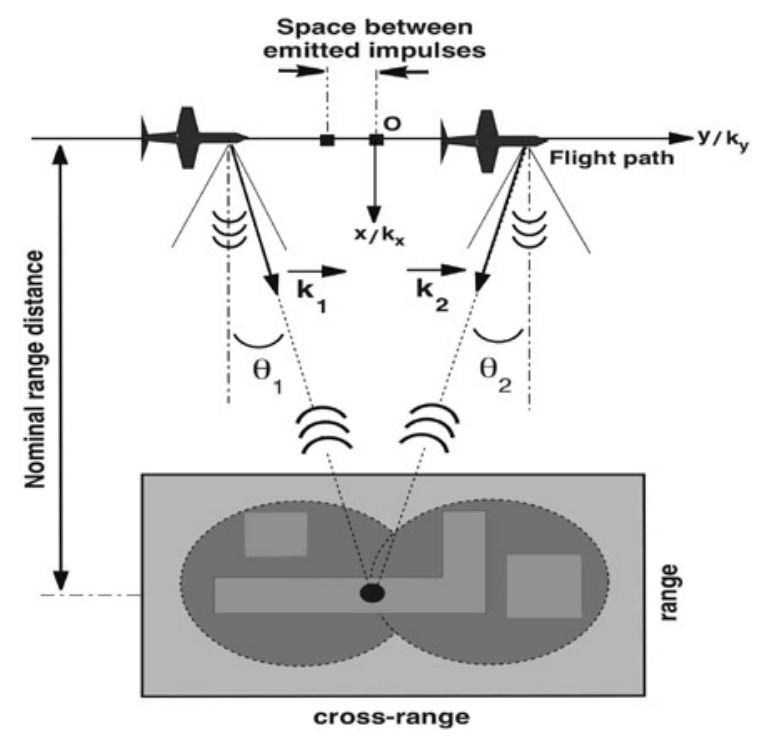

Fig. 1 Illustration of a scatterer irradiated at two different aspect angles in non-squintmode SAR imaging

amplitude dependence on aspect for localised scatterers such as trihedrals and corners is a slowly varying function modelled with a damped exponential function [8]. Third, the angle-dependent amplitude of distributed reflectors such as flat plates and dihedrals is dominated by a sinclike term as predicted by the physical optics far-field scattering theory [8]. By combining these dependences, the complex amplitude $\alpha_{i}(f, \theta)$ can be written in a single expression as follows

$$
\begin{aligned}
\sigma_{i}(f, \theta)= & A_{i}\left(\frac{j f}{f_{c}}\right)^{\alpha_{i}} \sin c\left(k L_{i} \sin \left(\theta-\theta_{i}\right)\right) \\
& \times \exp \left(-2 \pi f \gamma_{i} \sin (\theta)\right)
\end{aligned}
$$

where $A_{i}$ is a complex scalar, $L_{i}$ and $\theta_{i}$ the length and the orientation of the $i$ th scatterer, respectively, and $\gamma_{i}$ a parameter with no physical interpretation. The parameters $L_{i}$ and $\gamma_{i}$ are zero for localised and distributed scatterers, respectively. The model in (3) combined with (4) provides a concise physically relevant description of measured scattering for use in target recognition, identification and discrimination.

Referring to the model in (3), we suggest the formation of an image that depends on the frequency and the aspect, that is, one that depends on the wave vector $\boldsymbol{k}$. This image will be written $I(\boldsymbol{r}, \boldsymbol{k})$ and called an hyperimage because its dimension is the product of the dimension of the vector $r$ by the dimension of the vector $\boldsymbol{k}$. This hyperimage which provides a rich description of target scattering is not accessible from conventional Fourier-based imaging techniques and the construction needs a more advanced and appropriated analysis such as time-frequency methods. Among these methods, we propose to apply the multidimensional continuous wavelet transform (MCWT) in SAR imaging to construct such a hyperimage.

\section{Construction of hyperimages using the MCWT}

Before introducing the MCWT, we present the general construction of hyperimages from the time-frequency analysis. To do so, let us recall that the radar cross-section (RCS) of a target is defined as the squared modulus of the backscattering signal $H(\boldsymbol{k})$

$$
\sigma(\boldsymbol{k})=|H(\boldsymbol{k})|^{2}
$$

and is expressed in square meters [9]. The RCS is a function of the wavenumber $\boldsymbol{k}$, that is, the RCS depends on the variables $(f, \theta)$ and characterises the quantity of energy diffused by the target at the frequency $f$ and aspect angle $\theta$. Thus, the RCS provides in the $f-\theta$ plane, the distribution of the energy diffused by the scatterers. Unfortunately, the RCS provides no information concerning the position of these scatterers. To overcome this limitation, the timefrequency analysis has been introduced in SAR imaging to access to the energy distribution of the scatterers and also to their position. These two pieces of information can be obtained by constructing hyperimages $I_{\mathrm{H}}(\boldsymbol{r}, \boldsymbol{k})$ as hermitian and bilinear forms of the backscattering signal $H(\boldsymbol{k})$ [10]

$$
I_{\mathrm{H}}(\boldsymbol{r}, \boldsymbol{k})=\iint K\left(\boldsymbol{k}_{1}, \boldsymbol{k}_{2} ; \boldsymbol{r}, \boldsymbol{k}\right) H\left(\boldsymbol{k}_{1}\right) H^{*}\left(\boldsymbol{k}_{2}\right) \mathrm{d} \boldsymbol{k}_{1} \mathrm{~d} \boldsymbol{k}_{2}
$$

The term on the right of (6) is, in fact, a generalisation of the RCS formulation in (5) and $I_{\mathrm{H}}(\boldsymbol{r}, \boldsymbol{k})$ is an energy distribution in the hyperplane $(\boldsymbol{r}, \boldsymbol{k})$. The kernel $K$ is supposed to be hermitian and it can be determined with some physical constraints made on the distribution $I_{\mathrm{H}}(\boldsymbol{r}, \boldsymbol{k})[11,12]$; for example, one can impose that $I_{\mathrm{H}}(\boldsymbol{r}, \boldsymbol{k})$ should be positive in order that its integral on some surface $\mathcal{D}$ can be interpreted as the RCS contribution $\sigma_{\mathcal{D}}(\boldsymbol{k})$ of all the reflectors contained in $\mathcal{D}$

$$
\sigma_{\mathcal{D}}(\boldsymbol{k})=\int_{\mathcal{D}} I_{\mathrm{H}}(\boldsymbol{r}, \boldsymbol{k}) \mathrm{d} \boldsymbol{r}
$$

\subsection{The MCWT, a particular method for constructing hyperimages}

Let $\phi(k, \theta)$ be a mother wavelet supposed to represent the backscattering signal of a reference target localised around the position $\boldsymbol{r}=\mathbf{0}$. This target is supposed to diffuse mainly in the direction $\theta=0$ and at the frequency $f$ so that $k=2 f / c=1$. In other words, $\phi(k, \theta)$ is a 2D-function localised around $(k, \theta)=(1,0)$.

A family of wavelets $\Psi_{\boldsymbol{r}_{0}, \boldsymbol{k}_{0}}(\boldsymbol{k})$ can be generated from the mother wavelet $\phi(k, \theta)$ by rotation $\mathcal{R}_{\theta_{0}}$, translation $\boldsymbol{r}_{0}$ and contraction with the scale factor $1 / k_{0}$ according to $[9,11]$

$$
\begin{aligned}
\Psi_{\boldsymbol{r}_{0}, \boldsymbol{k}_{0}}(\boldsymbol{k}) & =\frac{1}{k_{0}} \mathrm{e}^{-2 i \pi \boldsymbol{k} \cdot \boldsymbol{r}_{0}} \phi\left(\frac{1}{k_{0}} \mathcal{R}_{\theta_{0}}^{-1} \boldsymbol{k}\right) \\
& =\frac{1}{k_{0}} \mathrm{e}^{-2 i \pi \boldsymbol{k} \cdot \boldsymbol{r}_{0}} \phi\left(\frac{k}{k_{0}}, \theta-\theta_{0}\right)
\end{aligned}
$$

The wavelet coefficients $C_{\mathrm{H}}\left(\boldsymbol{r}_{0}, \boldsymbol{k}_{0}\right)$ are introduced as the scalar product between the backscattering signal $H$ and each element $\Psi_{\boldsymbol{r}_{0}, \boldsymbol{k}_{0}}$ of the wavelet basis

$$
\begin{aligned}
C_{\mathrm{H}}\left(\overrightarrow{\boldsymbol{r}}_{0}, \overrightarrow{\boldsymbol{k}}_{0}\right)= & \int H(\boldsymbol{k}) \Psi_{\boldsymbol{r}_{0}, \boldsymbol{k}_{0}}^{*}(\boldsymbol{k}) \mathrm{d} \boldsymbol{k} \\
= & \int_{0}^{2} \mathrm{~d} \theta \int_{0}^{+\infty} k H(k, \theta) \\
& \times \frac{1}{k_{0}} \mathrm{e}^{j 2 \pi \boldsymbol{k} \cdot \boldsymbol{r}_{0}} \boldsymbol{\phi}^{*}\left(\frac{k}{k_{0}}, \theta-\theta_{0}\right) \mathrm{d} k \mathrm{~d} \theta
\end{aligned}
$$

From the general form of hyperimages defined in (6), a particular solution can be proposed on the basis of wavelet analysis. This solution is expressed as the squared modulus of the wavelet coefficients divided by the 
admissibility wavelet coefficient $A_{\phi}[11]$

$$
I_{\mathrm{H}}(\boldsymbol{r}, \boldsymbol{k})=\frac{1}{A_{\phi}}\left|C_{\mathrm{H}}(\boldsymbol{r}, \boldsymbol{k})\right|^{2}
$$

where $A_{\phi}$ is defined as

$$
A_{\phi}=\int \frac{|\phi(\boldsymbol{k})|^{2}}{k^{2}} \mathrm{~d} \boldsymbol{k}<\infty
$$

The kernel $K$ introduced in (6) which corresponds to the solution proposed in (9) is

$$
\begin{aligned}
K\left(\boldsymbol{k}_{1}, \boldsymbol{k}_{2}, \boldsymbol{r}, \boldsymbol{k}\right)= & \frac{1}{A_{\phi}} \frac{k_{1} k_{2}}{k^{2}} \mathrm{e}^{j 2 \pi\left(\boldsymbol{k}_{1}-\boldsymbol{k}_{2}\right) \cdot r} \\
& \times \phi^{*}\left(\frac{k_{1}}{k}, \theta_{1}-\theta\right) \phi\left(\frac{k_{2}}{k}, \theta_{2}-\theta\right)
\end{aligned}
$$

The reconstruction property allows to recover the signal with the knowledge of its wavelet coefficients

$$
H(\boldsymbol{k})=\frac{1}{A_{\phi}} \int \mathrm{d} \boldsymbol{r}_{0} \int C_{\mathrm{H}}\left(\boldsymbol{r}_{0}, \boldsymbol{k}_{0}\right) \Psi_{\boldsymbol{r}_{0}, \boldsymbol{k}_{0}}(\boldsymbol{k}) \mathrm{d} \boldsymbol{k}_{0}
$$

\subsection{Interpretation of the hyperimage $I(\boldsymbol{r}, \boldsymbol{k})$}

Let us rewrite $I(\boldsymbol{r}, \boldsymbol{k}) \equiv I(x, y ; f, \theta)$. For each reflector located at $\boldsymbol{r}_{0}=\left(x_{0}, y_{0}\right)$, we can extract its energetic feature $I\left(x_{0}, y_{0} ; f, \theta\right)$ as a function of frequency $f$ and aspect angle $\theta[12]$.

To analyse this 4D structure, a visual display interface called i4d [13] has been developed and allows to carry out an interactive and dynamic analysis. Figs. $3 a$ and $b$ describes the interface i4d that consists in selecting a pixel located at $\left(x_{0}, y_{0}\right)$ in the plane $(x, y)$, and visualising its corresponding energy response $I\left(x_{0}, y_{0} ; f, \theta\right)$. In Fig. $3 b$, the initial SAR image $I(x, y)$ is displayed inside the $x-y$ plane (left plot), in order that the user be sure that the pixel he selects corresponds to a reflector belonging to the object to be analysed. On two sides of the image plane, there are sliders that allow to select a pixel in the $x-y$ plane. The above and below right figures represent the energy response of the selected pixel, in $2 \mathrm{D}$ and $3 \mathrm{D}$, respectively.

\subsection{Wavelet parameters and resolutions}

In this paper, we do not tackle the question of the mother wavelet choice. Actually, any mother wavelet (Klauder, Mexican hat, ...) that is well localised at $(k, \theta)=(1,0)$ is suitable for analysing the SAR images. One of the more famous wavelets is the gaussian's wavelet. For our analysis, we have chosen the bi-dimensional gaussian, defined as

$$
\phi(k, \theta)=Y(k) \mathrm{e}^{-(k-1)^{2} / \sigma_{k}^{2}} \mathrm{e}^{-\theta^{2} / \sigma_{\theta}^{2}}
$$

where $Y(k)$ is the Heaviside distribution defined as

$$
Y(k)= \begin{cases}0 & \text { if } \mathrm{k} \leq 0 \\ 1 & \text { else }\end{cases}
$$

Parameters $\left(\sigma_{k}, \sigma_{\theta}\right)$ characterise the frequency and angular resolutions, respectively: indeed, they determine the frequency and angular bandwidths $\delta k$ and $\delta \theta$ of mother wavelet $\phi(k, \theta)$ and so control the frequency and angular spread of the wavelets $\Psi_{r_{0}, \boldsymbol{k}_{0}}(\boldsymbol{k})$, respectively. Bandwidths $\delta k$ and $\delta \theta$ are defined as the ' $3 \mathrm{~dB}$ pass-band' (also called 'mean-square bandwidth') [14]. This $3 \mathrm{~dB}$ pass-band is defined as follows:
- By denoting $k_{1}=1$ the reference wavenumber, $3 \mathrm{~dB}$ passband $\delta k$ is defined according to

$$
\frac{\left|\phi\left(k_{1} \pm(\delta k / 2), \theta\right)\right|^{2}}{\left|\phi\left(k_{1}, \theta\right)\right|^{2}}=\frac{1}{2}
$$

- Angular bandwidth $\delta \theta$ is defined the same way with the reference angle $\theta_{1}=0$.

In our applications, $\delta \theta$ is equal to $15 \%$ of the full angular aperture $\Delta \theta$. Bandwidth $\delta k$ has been determined so that the wavelets localised at $k_{\mathrm{c}}=2 f_{\mathrm{c}} / c\left(f_{\mathrm{c}}\right.$ is the central frequency) have a spread equal to $15 \%$ of $\Delta k=2 B / c$ ( $B$ is the frequency bandwidth).

\subsection{Algorithm for computing the MCWT}

To describe the algorithm which allows to extend the MCWT in SAR imaging, let us recall the mathematical expression of the wavelet transform in (9) by considering a real mother wavelet $\phi$

$$
\begin{aligned}
C\left(\boldsymbol{r}_{0}, \boldsymbol{k}_{0}\right)= & \int_{0}^{2 \pi} \int_{0}^{+\infty} k H(k, \theta) \Psi_{\boldsymbol{r}_{0}, \boldsymbol{k}_{0}}(k, \theta) \mathrm{d} \boldsymbol{k} \mathrm{d} \theta \\
= & \int_{0}^{2 \pi} \int_{0}^{+\infty} k H(k, \theta) \frac{1}{k_{0}} \phi\left(\frac{k}{k_{0}}, \theta-\theta_{0}\right) \\
& \times \exp \left(j 2 \pi\left[k x_{0} \cos \theta+k y_{0} \sin \theta\right]\right) \mathrm{d} \boldsymbol{k} \mathrm{d} \theta
\end{aligned}
$$

With a change of variables $\left(k_{x}, k_{y}\right)=(k \cos \theta, k \sin \theta)$, the second member of the previous equation can be expressed as

$$
\begin{aligned}
& C\left(\boldsymbol{r}_{0}, \boldsymbol{k}_{0}\right) \\
& =\int_{\mathbb{R}^{2}} H\left(k_{x}, k_{y}\right) \frac{1}{k_{0}} \phi\left(\frac{\sqrt{k_{x}^{2}+k_{y}^{2}}}{k_{0}}, \arctan \left(\frac{k_{y}}{k_{x}}\right)-\theta_{0}\right) \\
& \quad \times \exp \left(j 2 \pi\left[k_{x} x_{0}+k_{y} y_{0}\right]\right) \mathrm{d} k_{x} \mathrm{~d} k_{y} \\
& =\mathcal{F}_{\substack{k_{x} \rightarrow x_{0} \\
k_{y} \rightarrow y_{0}}}^{-1}\left[H\left(k_{x}, k_{y}\right) \Phi_{k_{0}, \theta_{0}}\left(k_{x}, k_{y}\right)\right] \\
& \quad
\end{aligned}
$$

where

$$
\Phi_{k_{0}, \theta_{0}}\left(k_{x}, k_{y}\right)=\frac{1}{k_{0}} \phi\left(\frac{\sqrt{k_{x}^{2}+k_{y}^{2}}}{k_{0}}, \arctan \left(\frac{k_{y}}{k_{x}}\right)-\theta_{0}\right)
$$

Hence, referring to the relation (11), the wavelet coefficients can be calculated by using the bi-dimensional Fourier transform. The steps of the algorithm are developed in the following paragraph.

2.4.1 Steps of the algorithm: First, let us construct the spectrum $H\left(k_{x}, k_{y}\right)$ by performing the bi-dimensional Fourier transform of the complex-valued image $I(x, y)$

$$
\begin{gathered}
H\left(k_{x}, k_{y}\right)=\mathcal{F}_{x \rightarrow k_{x}}[I(x, y)] \\
y \rightarrow k_{y}
\end{gathered}
$$

then, for each couple of wavenumber-angle values $\left(k_{i}, \theta_{j}\right)$,

(1) Construct the analysis window localised around $\left(k_{i}, \quad \theta_{j}\right), \quad \phi_{k_{i}, \theta_{j}}(k, \theta)=\left(1 / k_{i}\right) \phi\left(k / k_{i}, \theta-\theta_{j}\right) \quad$ using the 
variables $\left(k_{x}, k_{y}\right)$

$$
\Phi_{k_{i}, \theta_{j}}\left(k_{x}, k_{y}\right)=\frac{1}{k_{i}} \phi\left(\frac{\sqrt{k_{x}^{2}+k_{y}^{2}}}{k_{i}}, \arctan \left(\frac{k_{y}}{k_{x}}\right)-\theta_{j}\right)
$$

(2) Make the product: $\Pi_{k_{i}, \theta_{j}}\left(k_{x}, k_{y}\right)=\boldsymbol{H}\left(k_{x}, k_{y}\right) \Phi_{k_{i}, \theta_{j}}\left(k_{x}, k_{y}\right)$ (3) The wavelet coefficient $C_{\mathrm{H}}\left(\boldsymbol{r}_{0}, \boldsymbol{k}_{i j}\right)$ is obtained by bi-dimensional inverse fourier transform on the 2D-function $\Pi_{k_{i}, \theta_{j}}\left(k_{x}, k_{y}\right)$. To compute them efficiently, we use the fast Fourier transform (FFT)

$$
\begin{gathered}
C_{\mathrm{H}}\left(\boldsymbol{r}_{0}, \boldsymbol{k}_{i j}\right)=\mathrm{FFT}_{k_{x}} \rightarrow x_{0}\left[\Pi_{k_{i}, \theta_{j}}\left(k_{x}, k_{y}\right)\right] \\
k_{y} \rightarrow y_{0}
\end{gathered}
$$

Once the wavelet coefficients are calculated for all the couples of wavenumber-angle values $\left(k_{i}, \theta_{j}\right)$, the hyperimage $I_{\mathrm{H}}\left(\boldsymbol{r}_{0}, \boldsymbol{k}_{0}\right)$ is defined by the squared modulus of the wavelet coefficients divided by the admissibility coefficient $A_{\phi}$

$$
I_{\mathrm{H}}\left(\boldsymbol{r}_{0}, \boldsymbol{k}_{0}\right)=\frac{1}{A_{\phi}}\left|C_{H}\left(\boldsymbol{r}_{0}, \boldsymbol{k}_{0}\right)\right|^{2}
$$

2.4.2 Complexity of the algorithm: The approximate complexity of the algorithm can be expressed in terms of the number of FFT performed in step 3, (13). Assuming a complex-valued image $I(x, y)$ of $\left(N_{x} \times N_{y}\right)$ samples and referring to the Fourier relation in (12), the spectrum $H\left(k_{x}, k_{y}\right)$ will be on a grid of $\left(N_{k_{x}} \times N_{k_{x}}\right)$ samples with $N_{k_{x}}=N_{x}$ and $N_{k_{v}}=N_{y}$. We denote $\left(N_{k_{0}}, N_{\theta_{0}}\right)$ the numbers of values $k_{0}=2 f_{0} / c$ (where $f_{0}$ represents a frequency value) and aspects $\theta_{0}$.

Consequently, by referring to the relation in (13), we have to deal with

$$
N_{k_{0}} N_{\theta_{0}}\left(N_{k_{x}} \text { FFT [of } N_{k_{y}} \text { samples] }+N_{k_{y}} \text { FFT [of } N_{k_{x}} \text { samples] }\right)
$$

In our SAR applications, the values used are typically

$$
N_{x}=N_{k_{x}}=512, \quad N_{y}=N_{k_{y}}=512, \quad N_{k_{0}}=N_{\theta_{0}}=40
$$

The following section is devoted to the use of the hyperimage $I_{\mathrm{H}}$ for target discrimination in SAR imaging. Indeed, by fixing the first and the second dimension of the hyperimage, we access to $I_{\mathrm{H}}\left(x_{i}, y_{i} ; f, \theta\right)$ which is the energy distribution of the scatterer localised at $\left(x_{i}, y_{i}\right)$. Referring to the scattering model given by (3) and (4) which takes into account the scatterers' geometry, our aim is to distinguish in a SAR image, one object from other ones by exploiting the energy distributions of all scatterers contained in this image.

\section{Discriminating different kinds of object using the wavelet analysis}

The ONERA airborne [15] SAR system, called RAMSES, allows to process real data collected in different frequency ranges (X-band, Ku-band, P-band, ...) for the formation of high-resolution SAR images. Therefore, the target discrimination method proposed in this paper can be potentially tested on many targets. In this paper, we analyse four SAR images and we test the target discrimination technique on different objects included in these images.

\subsection{Highlighting the reflectors' energy distributions}

In this paragraph, we highlight the frequency and aspect angle dependencies of real scatterers by analysing four SAR images. The analysis of the first two images is also described [12, 16, 17]. These images come from the same measurement campaign with a fixed objects and targets orientation on the ground. So it has not been possible to test the robustness of this technique for different object orientations. In the examples presented below, the central frequency is $f_{\mathrm{c}}=14.2 \mathrm{GHz}$ (Ku-band), the bandwidth is $B=900 \mathrm{MHz}$ (that corresponds to a relative bandwidth $\left.B_{\mathrm{r}}=B / f_{\mathrm{c}}=6 \%\right)$ and the full angular aperture is $\Delta_{\theta}=3^{\circ}$. Hence, the analysed target or object is seen only for a subset of wavenumbers $\boldsymbol{k}$. So, it implies that the wavelet analysis can give a full description of the scatterers RCS only in this range of measure. This fact limits the possibility of discriminating the target for any orientation on the ground. The following is the description of the four analysed SAR images:

- The first image is composed of a pair of tanks, stairs (on the left) and a footbridge laid along the vertical (see area (a) in Fig. 2). Thanks to the interface i4d described in the Fig. 3, we have selected different pixels (in the $x-y$ plane) localised on the tanks, the footbridge and the stairs, and we have visualised the corresponding energy response in the $f-\theta$ plane: we have carried the responses of these pixels forward to the Fig. 4. Observing Fig. 4, we can notice that the tanks' reflectors have similar responses. Also, on the footbridge and the stairs, the analysed scatterers' responses are almost identical: the aspect behaviours seem to be dominated by a gaussian or a sinclike as predicted by the model in (4) for distributed reflectors. However, these reflectors have energetic characteristics which differ from the ones of the reflectors on the pair of tanks. Considering the previous experimental observations, our aim has been to separate the pair of tanks from the combination of the footbridge and the stairs by exploiting the energetic characteristics of all the reflectors in the image.

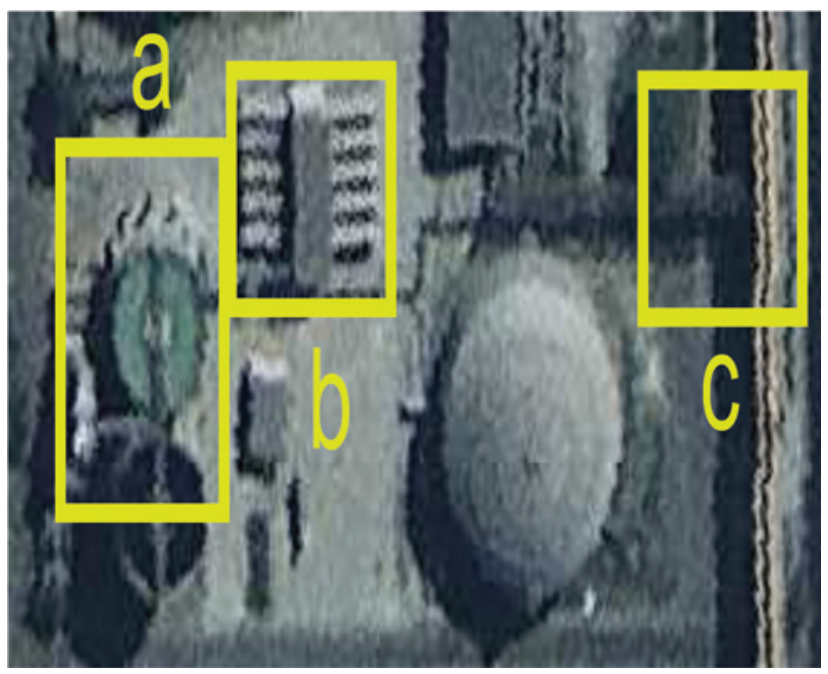

Fig. 2 Aerial photography containing three of the four images analysed

$a$ Couple of tanks with a footbridge (along the vertical) and stairs (on the left)

$b$ Lateral buildings attached to a big one

$c$ Building (along the horizontal) and pipe (along the vertical) 

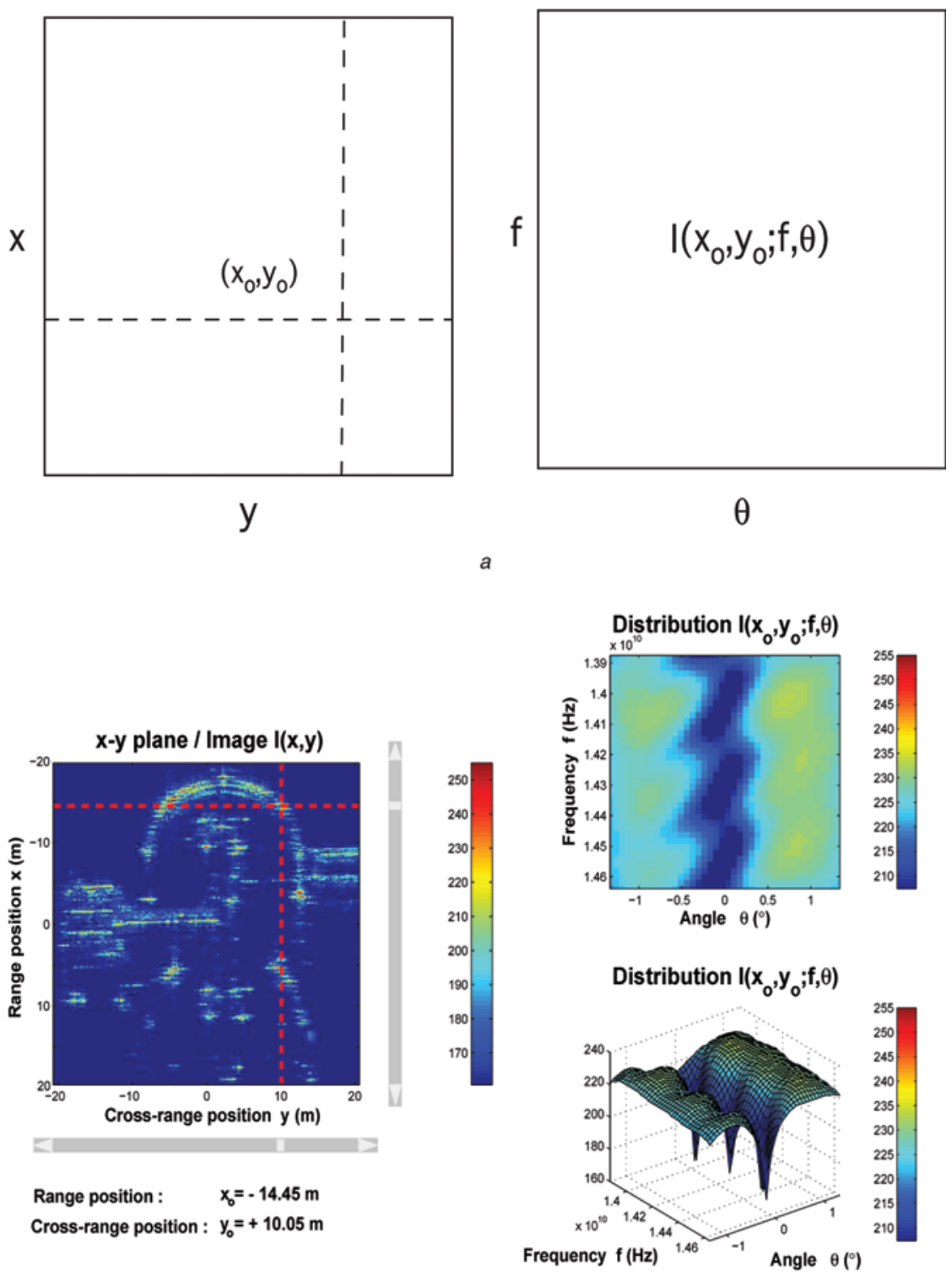

$b$

Fig. 3 Visual interface i4d to visualise the energy response of a given reflector

$a$ Presentation of i4d

$b$ Visualisation of the response of a reflector belonging to a tank

- The second study concerns a warehouse: lateral buildings are attached to a big one on both sides (see area (b) in Fig. 2). Again, with the help of the interface i4d, we have selected different pixels (in the $x-y$ plane) localised on the lateral buildings' roofs and on the central building's ridge, and we have visualised the corresponding energy response in the $f-\theta$ plane: we have carried the responses of these pixels forward to Fig. 5. On the lateral buildings' roofs, the responses of the analysed reflectors are almost identical and again the aspect-varying responses seem to follow the prediction of the model in (4) in the case of distributed reflectors. However, the responses of these reflectors differ clearly from the responses of the scatterers on the central building's ridge that seem more random. At the sight of the results presented in the Fig. 5, the work has consisted in distinguishing the roof of the lateral buildings from the ridge of the central one thanks to the knowledge of the energetic characteristics of these two types of object.
Following the above step, we have visualised the response of different scatterers in the following two SAR images, and the observations that we have made in terms of scatterers' energy response, have suggested us to discriminate some target or other.

- Area (c) in Fig. 2 represents a building laid along the horizontal, and a pipe along the vertical: our aim has been to extract the building and subtract the pipe.

- The last image analysed is a building with objects that we are not able to identify, on the roof (see Fig. 9): the purpose has been to extract one ridge of the building and subtract the objects on the roof.

The observations presented in the Figs. 4 and 5 reveal different classes of reflectors at the sight of their frequency and behaviours. Each class of reflectors corresponds to a specific type of object. These observations lead us to propose an algorithm whose purpose is to discriminate a 


\section{Energy responses of scatterers on the}

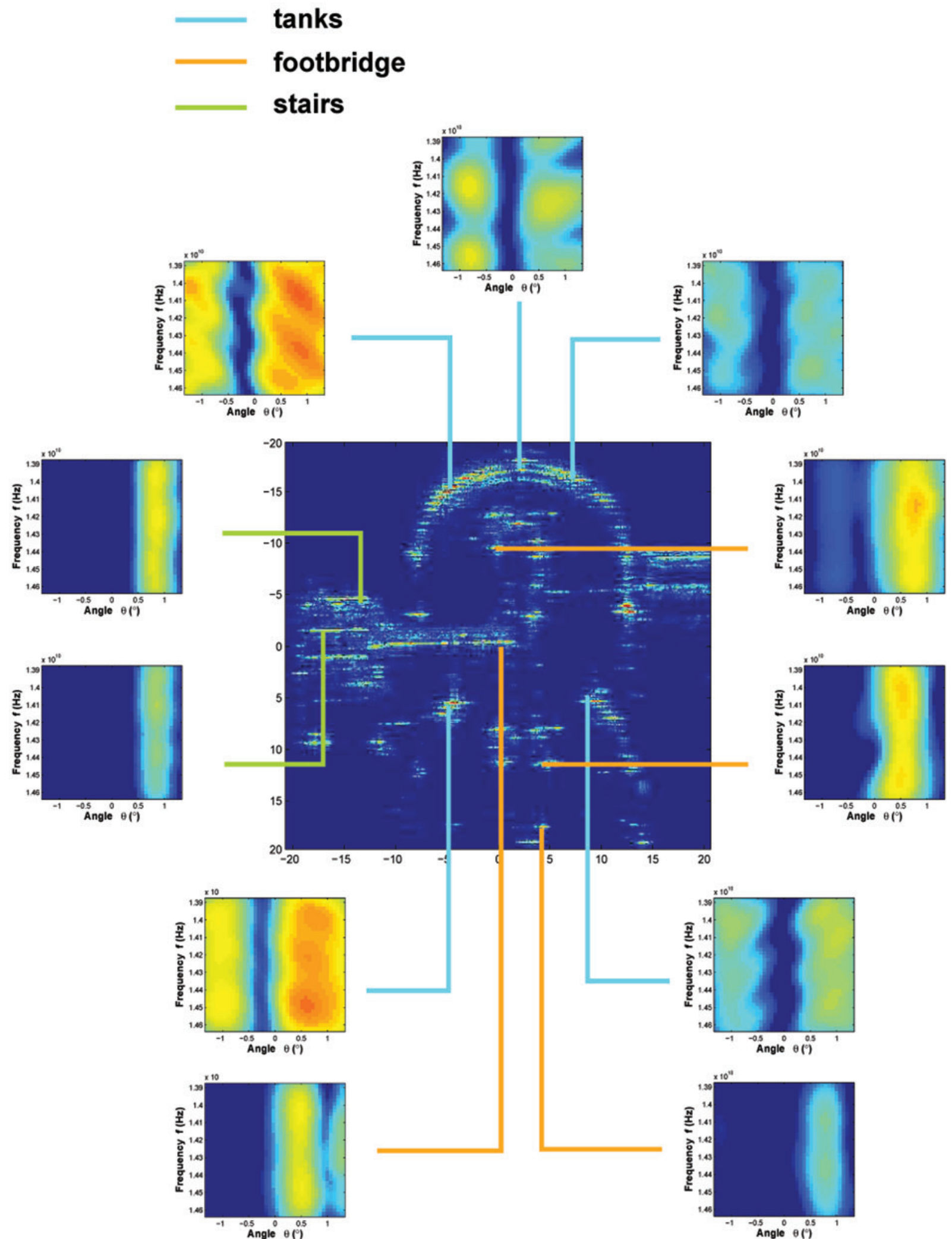

Fig. 4 Energetic characteristics of some scatterers localised on the tanks and the combination of the footbridge and the stairs

type of object in a SAR image by exploiting the energy distributions of all the scatterers.

\subsection{Development of an algorithm for discriminating different types of object}

The object type discrimination algorithm (OTDA) that we propose in this section makes two hypotheses:
- One scatterer which belongs to an object is supposed to have a frequency and aspect behaviour similar to a scatterer of another reflector localised in this object.

- The energy distribution of one reflector belonging to a first object is assumed to differ from the distribution of another reflector localised on a second object with different geometry and/or orientation relative to the former object. 


\section{Energy responses of scatterers on the}

\section{central building's ridge}

\section{_ lateral buildings' roofs}

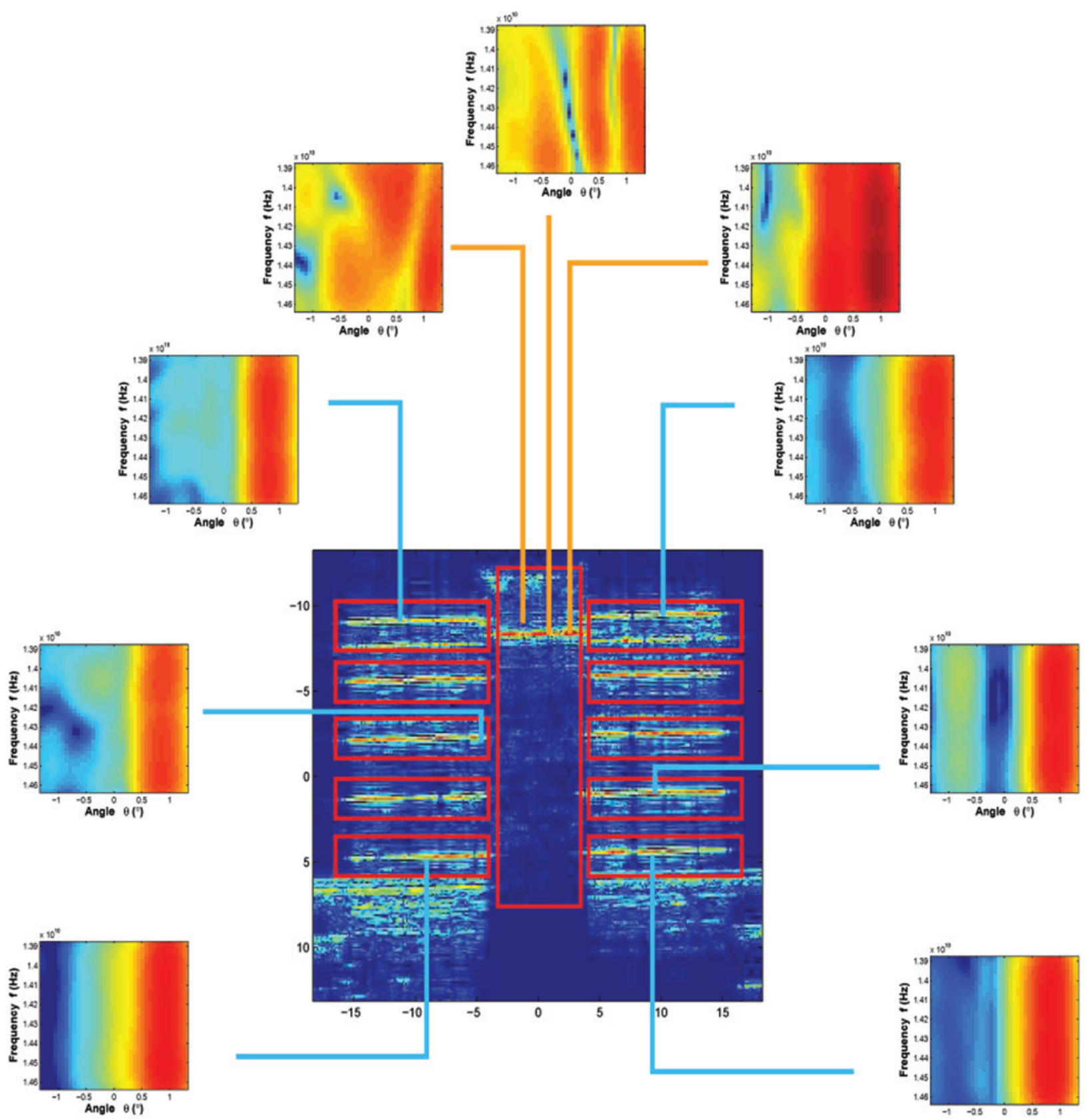

Fig. 5 Energetic characteristics of some scatterers localised on the lateral buildings' roofs and on the central building's ridge

The basic idea we propose is to select among all the scatterers' energy distributions, one energy distribution susceptible to be characteristic of the type of object to be discriminated: this distribution becomes a reference. Then, the purpose is to identify the scatterers in the image which have similar distributions to the reference.

Before introducing the OTDA, a threshold is applied on all the energy distributions since we suppose that the energetic characteristics of a scatterer remain within a confined dynamic of its energy distribution. Consequently, we choose a dynamic $-\Delta_{\mathrm{dB}}$ identical for all the distributions: the threshold, for each distribution, is placed at $-\Delta_{\mathrm{dB}}$ from the maximum of this distribution. To do so, we sweep across the $x-y$ plane and for each pixel localised in $\left(x_{i}, y_{j}\right)$, we determine the maximum value of the corresponding distribution $I\left(x_{i}, y_{j} ; f, \theta\right): I_{i, j}^{\max }=\max _{f, \theta}\left[I\left(x_{i}, y_{j} ; f, \theta\right)\right]$ then we calculate the corresponding threshold value: $T_{i, j}=I_{i, j}^{\max }$ $\left.10^{-\left(\Delta_{\mathrm{dB}} / 20\right.}\right)$, finally we set to zero all the energy values that are smaller than the threshold $T_{i, j}$

$$
\text { if } I\left(x_{i}, x_{j} ; f, \theta\right) \leq T_{i, j} \text {, then } I\left(x_{i}, x_{j} ; f, \theta\right)=0
$$

Once the threshold process is done, we execute the OTDA. The OTDA consists in selecting arbitrarily a scatterer on the target to be extracted, whose energy response seems to be characteristic of this target. The process for selecting the reflector is the following: by using the interface i $4 \mathrm{~d}$ (see Fig. $3 b$ ), we visualise the energy response of different 
pixels localised on the object, and we select a reflector whose energy response is similar to another responses that we have visualised and corresponding to reflectors on the target. Then, we correlate the high-energy response of the reflector selected, with the high-energy response of all the pixels in the image plane. The more the high-energy response of a pixel similar to the reference high-energy response, the larger the corresponding correlation coefficient value. The objective of the above method is that the large correlation coefficient values highlight the scatterers that compose the target to be discriminated. The steps of the OTDA are described as follows:

\section{Object type discrimination algorithm}

- Select a pixel on the object to be distinguished. By denoting $\left(x_{0}, y_{0}\right)$ the coordinates of the selected pixel, the energy distribution of this pixel $I_{0}(f, \theta) \equiv I\left(x_{0}, y_{0} ; f, \theta\right)$ is considered as a reference function.

- Calculate the following 2D-correlation function by introducing the reference distribution and the distribution of the other pixels in the image $\left\{I\left(x_{i}, x_{j} ; f, \theta\right) ;(i, j) \in\left[1, N_{x}\right] \times\right.$ $\left.\left[1, N_{y}\right]\right\}$

$$
C_{0}\left(x_{i}, y_{j}\right)=\frac{1}{\sqrt{E_{0}} \sqrt{E_{i, j}}} \int I_{0}(f, \theta) I_{i, j}(f, \theta) \mathrm{d} f \mathrm{~d} \theta
$$

where $\left(N_{x}, N_{y}\right)$ are the numbers of samples in slant-range and cross-range, respectively, we adopt the simplified notation $I_{i, j}(f, \theta) \equiv I\left(x_{i}, x_{j} ; f, \theta\right)$ and $E_{i, j}$ is defined as

$$
E_{i, j}=\int\left|I\left(x_{i}, y_{j} ; f, \theta\right)\right|^{2} \mathrm{~d} f \mathrm{~d} \theta
$$

Remark 1: This algorithm does not exploit the phase of the wavelet coefficients which contains information. Thus, it can be improved by introducing the complex wavelet coefficients in the correlation function [21] instead of the energy distribution: the $2 \mathrm{D}$ correlation function becomes

$$
\begin{aligned}
C_{\text {ref }}(i, j)= & \frac{1}{\sqrt{E_{\text {ref }}} \sqrt{E_{i, j}}} \\
& \times \int W\left(x_{\text {ref }}, y_{\text {ref }} ; f, \theta\right) W^{*}\left(x_{i}, y_{j} ; f, \theta\right) \mathrm{d} f \mathrm{~d} \theta
\end{aligned}
$$

where

$$
\begin{aligned}
E_{\text {ref }} & =\int\left|W\left(x_{\text {ref }}, y_{\text {ref }} ; f, \theta\right)\right|^{2} \mathrm{~d} f \mathrm{~d} \theta \\
E_{i, j} & =\int \mid W\left(x_{i}, y_{i} ; f, \theta\right)^{2} \mathrm{~d} f \mathrm{~d} \theta
\end{aligned}
$$

This substitution of the distributions by the wavelet coefficients is justified by the fact that the wavelet coefficient contains a phase information that is susceptible to discriminate the target. This idea is strengthened by the fact that the use of the complex wavelet coefficients in polarimetry and interferometry has allowed to improve the distinction of natural targets from artificial ones and the estimation of the target height, respectively [18-20]. We have noticed that the target discrimination method (involving the squared modulus of the wavelet coefficients) presented in this paper, which depends on the choice of the reference pixel, is not reliable in some cases. We have shown that exploiting not only the modulus but also the argument of the wavelet coefficients, can improve the detection of a certain class of reflectors. A first result stemming from the full exploitation of the wavelet coefficients can be found in the work of Tria et al. [21].

\section{Results in object type discrimination}

In this section, the purpose is to separate the tanks and the combination of the footbridge and the stairs in the first SAR image (see area (a) in the Fig. 2), distinguish the ridge of the central building from the roofs of the lateral buildings in the second image (see area (b) in Fig. 2), extract one ridge of the building and subtract the pipe in the third image (see area (c) in Fig. 2) and extract a ridge of the building in the fourth image (see Fig. 9).

\subsection{Separation of the tanks and the combination of the footbridge and the stairs}

To extract the tanks, we apply the algorithm by selecting in the image, a pixel whose energy distribution is susceptible to be characteristic of the tanks: this distribution is considered as the reference function. Then, we calculate the 2D-correlation function introduced in step 2 of the OTDA. Fig. $6 a$ represents this bi-dimensional function in
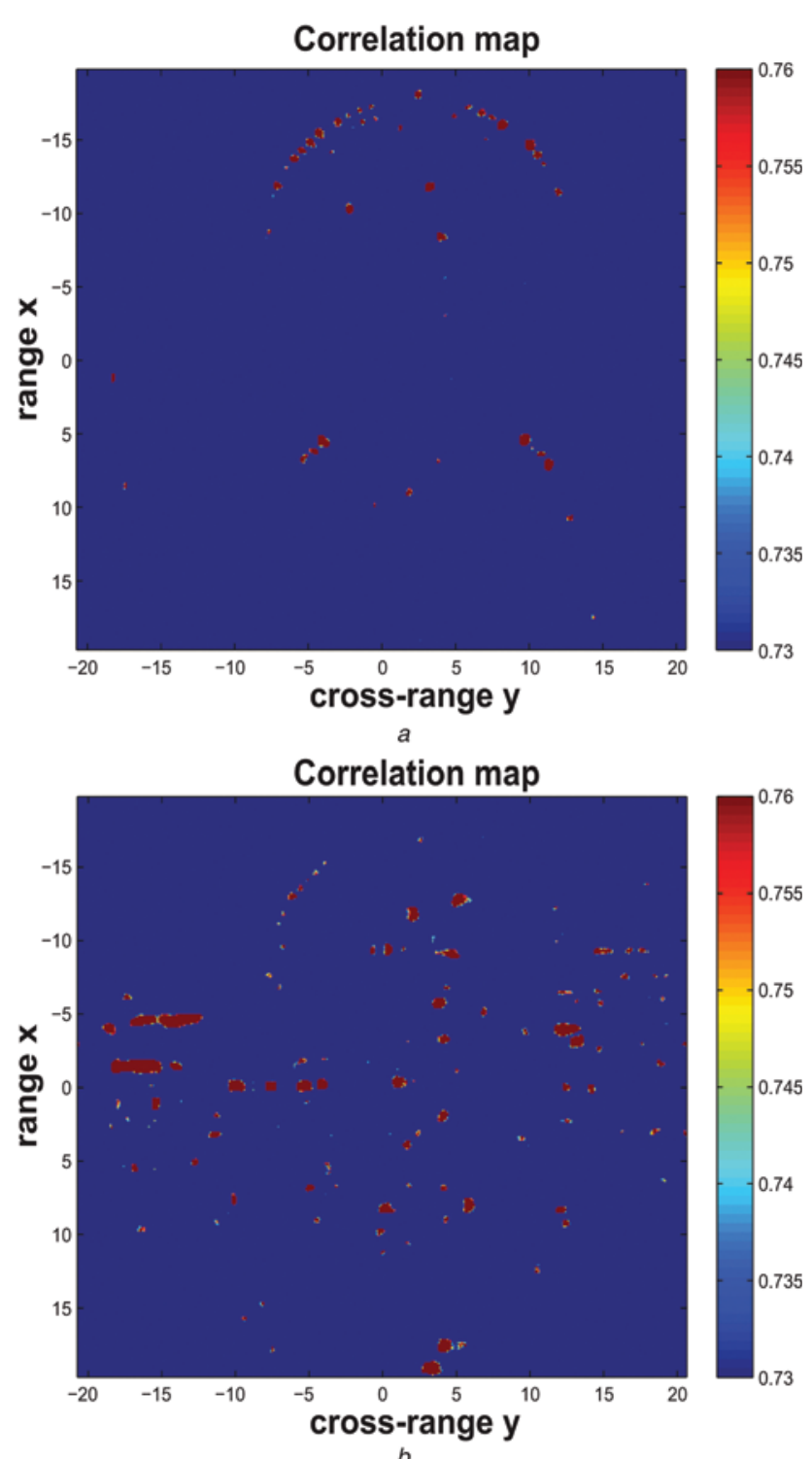

Fig. 6 Separation of the pair of tanks and the set composed of the footbridge (on the vertical) and the stairs (on the left)

$a$ Extraction of the tanks using the ODTA

$b$ Extraction of the set footbridge-stairs using the ODTA 

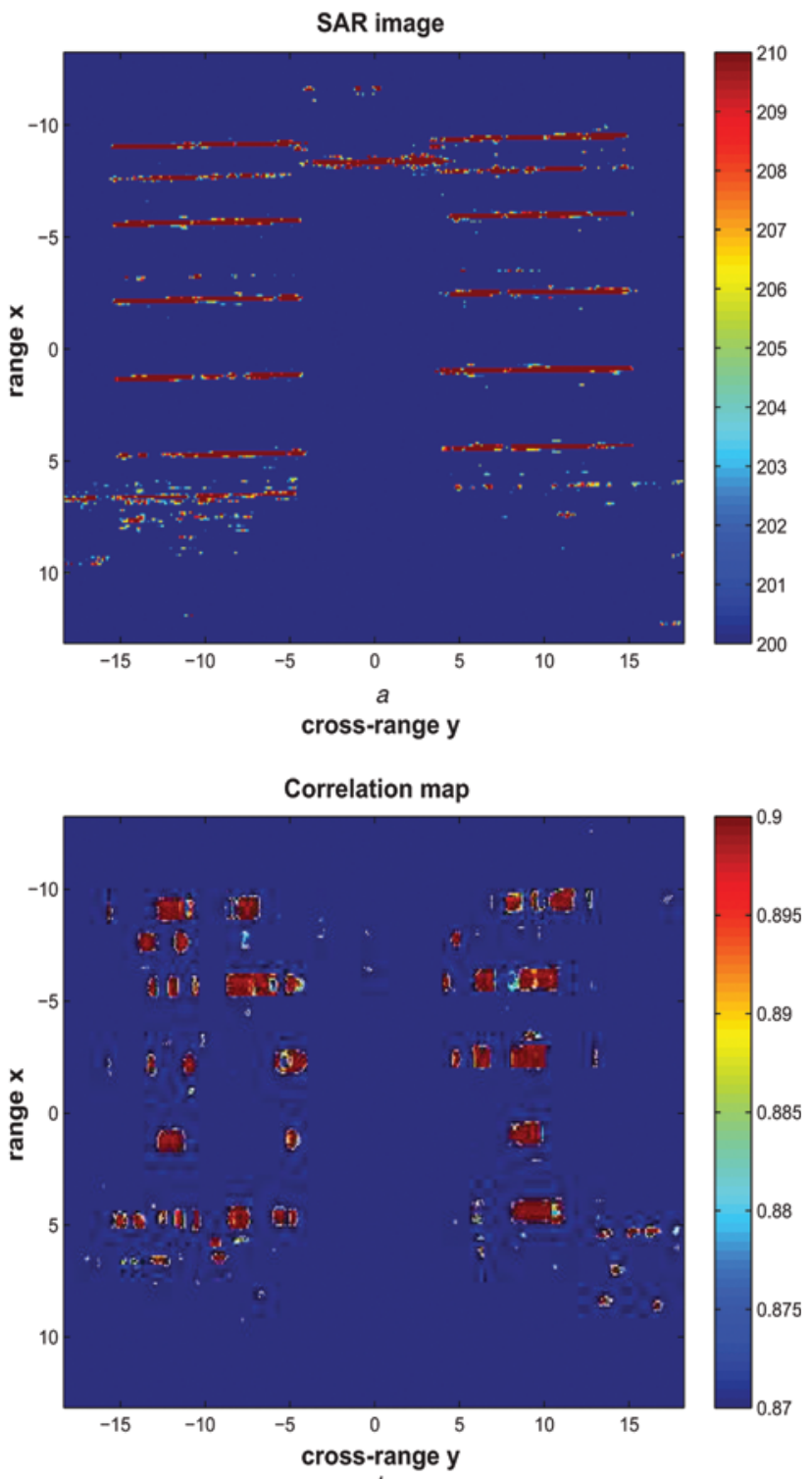

Fig. 7 Subtraction of the central building's ridge, whereas the lateral buildings' roof are extracted

$a$ Initial SAR image composed of lateral buildings attached to a big one $b$ Extraction of the lateral buildings using the ODTA

the $x-y$ plane (we call this representation 'correlation map': high- and low-correlation coefficients are represented by the light gray and dark gray colours respectively): we can notice that the high-correlation values correspond to scatterers belonging to the tanks. To discriminate the footbridge and the stairs, we proceed the same way by considering as the reference function, the energy distribution of one pixel situated on the footbridge. The correlation map is illustrated in Fig. $6 b$ : the footbridge and the stairs are discriminated.

\subsection{Subtraction of a building's ridge}

In this second study, our objective is to extract the roof of the lateral buildings and distinguish them from the ridge of the central one. More precisely, we want to subtract the second object. To do so, we execute the OTDA by considering the energy distribution of one pixel localised on the roof of one lateral building, as the reference function. In Fig. 7b, the correlation map is illustrated and compared with the initial SAR image, in Fig. 7a: the roof of the lateral
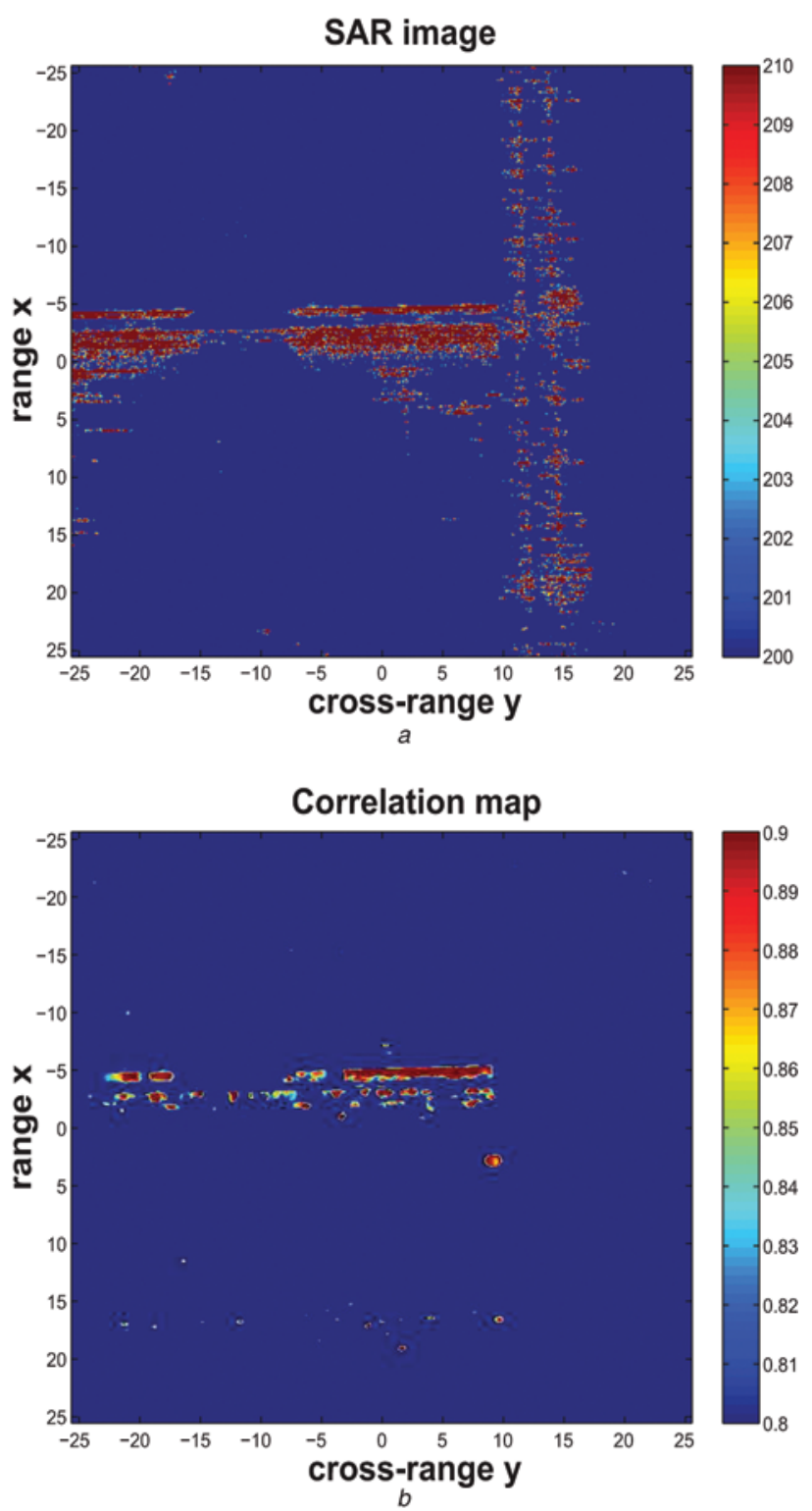

Fig. 8 Subtraction of the pipe, whereas a portion of the building is distinguished

$a$ Initial SAR image composed of a building (along the horizontal) and a pipe (along the vertical)

$b$ Extraction of a portion of the building, whereas the pipe is substracted, using the ODTA

buildings are discerned, whereas the ridge of the central building is not visible.

\subsection{Extraction of a portion of a building}

Fig. $8 a$ represents the initial SAR image composed of a building and a pipe. Our aim has been to extract a building's ridge and subtract the pipe, by correlating the high-energy response of all the pixels in the $x-y$ plane, with the high energy of one scatterer belonging to the building's ridge. The correlation map is illustrated in the Fig. $8 b$ : by comparing with Fig. $8 a$, we can see that the ridge of the building is clearly discriminated.

\subsection{Extraction of a building's ridge}

The last study concerns the building represented in the Fig. 9. The purpose has been to extract a ridge of the building and subtract the components on the roof. To do so, we 


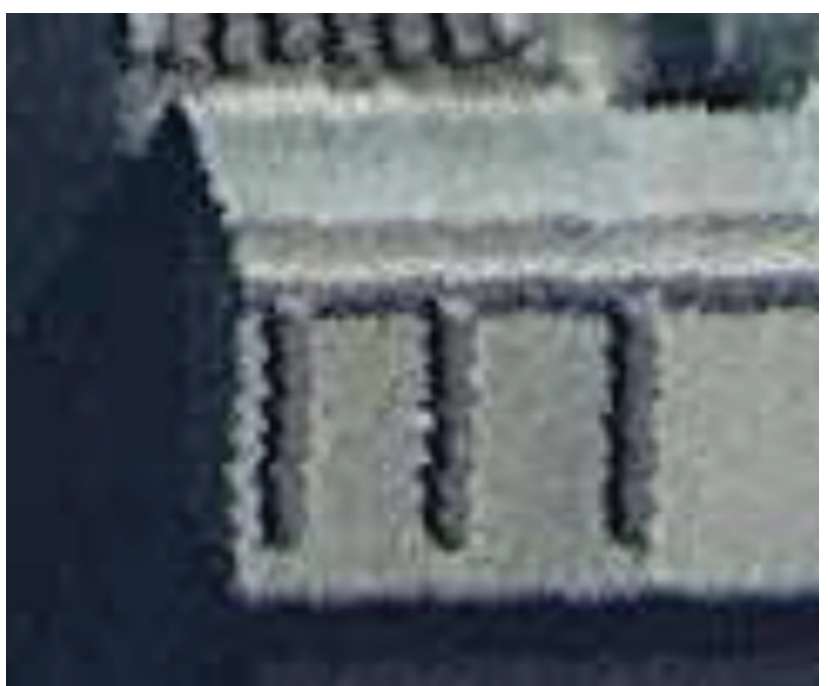

Fig. 9 Aerial photography corresponding to the fourth image analysed: a building seen from the air

have used the interface i4d to detect the energy characteristic response of the ridge localised around the range $x=-15 \mathrm{~m}$ (see Fig. 10a), and we have applied the ODTA in order that the high-correlation values correspond to scatterers belonging to the ridge to be distinguished. The correlation map is illustrated in Fig. $10 b$ and compared with the initial SAR image in the Fig. 10a: we can observe that the ridge is clearly extracted around the range position $x=-15 \mathrm{~m}$, the second ridge around the range $x=17 \mathrm{~m}$ is weakly discriminated and two other filiform components around $x=-17 \mathrm{~m}$ and $x=-12 \mathrm{~m}$ are also extracted. The component around the range $x=-17 \mathrm{~m}$ is actually the intersection between the floor and the building (see Fig. 9); the component around the range $x=-12 \mathrm{~m}$ is an edge of the straight object, laid along the horizontal (see Fig. 9). It is to notice the existence of a straight object laid along the horizontal around the range $x=2 \mathrm{~m}$ (see Fig. 10a), which is not present in the aerial photography representing the building (see Fig. 9).

\section{Conclusion and perspectives}

The following conclusions can be drawn from the experimental results stemming from the wavelet analysis and the OTDA. First, some reflectors analysed have an aspect-dependent response, which follows the prediction of the physical optics for distributed scatterers. Concerning the object discrimination, the results are encouraging since different objects have been extracted from the four images analysed. However, we can propose some modifications in the OTDA to improve the results. Instead of choosing a reference distribution in the analysed image, it should be more convenient to establish a bank of theoretical frequency and aspect varying responses corresponding to canonical objects (e.g. trihedral, dihedral, sphere, cylinder, flat plate and so on), each of which can be considered as a reference response.

In addition, we can propose to introduce in the $2 \mathrm{D}$ correlation function, a distribution resulting from the contribution of a neighbourhood $V_{i, j}$ around the pixel $\left(x_{i}, y_{j}\right)$

$$
I\left(V_{i, j} ; f, \theta\right)=\int_{V_{i, j}} I(x, y ; f, \theta) \mathrm{d} x \mathrm{~d} y
$$

instead of the distribution of one pixel localised at $\left(x_{i}, y_{j}\right)$, since the slant-range and cross-range resolutions degrade (in virtue of the Heisenberg's principle) by introducing
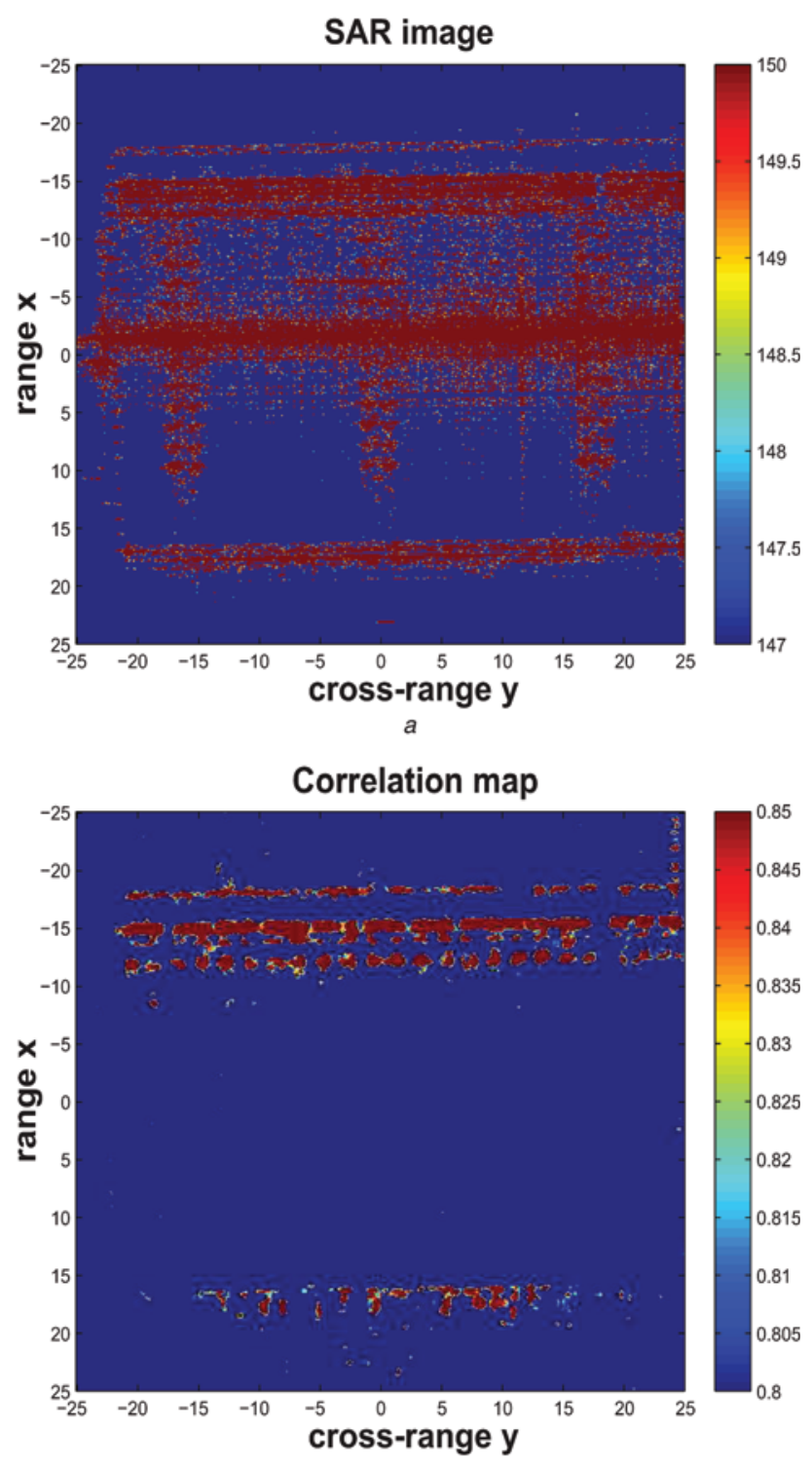

$b$

Fig. 10 Building's ridges, the intersection between the floor and the building, and an edge of an object on the roof are extracted, whereas the other components on the roof are subtracted

$a$ Initial SAR image of a building with longitudinal objects on the roof $b$ Extraction of the building's ridges, the intersection between the floor and the building, and an edge of an object on the roof

the wavelet analysis and consequently the reflector's position to be analysed is not known accurately. Here, the choice of the size of the window around the pixel localised at $\left(x_{i}, y_{j}\right)$, has to be discussed. Moreover, the wavelet analysis has to be extended to SAR images formed over wide angular apertures. For example, in X-band and in P-band, this aperture can reach $\Delta \theta=20^{\circ}$ and $\Delta \theta=120^{\circ}$, respectively, although the SAR images analysed in this paper have been formed over a small angular aperture $\Delta \theta=3^{\circ}$, which limits the target discrimination. This extension to wide apertures can be interesting to classify the objects according to their orientation. As well, it will be important to analyse images built with broad bandwidths in order to check whether the frequency-dependent behaviours of real scatterers follows the GTD prediction: for example, with the P-band, we have the possibility to work with relative bandwidth $B_{\mathrm{r}}=16 \%$, whereas our analysis have been made in $\mathrm{Ku}$-band with a relative bandwidth $B_{\mathrm{r}}=B / f_{\mathrm{c}}=6 \%$, which limits the target discrimination again. 
Finally, the aim of separating different types of object can be connected to a problem of sources classification: the scatterers (on the different types of object) can be identified as sources since they emit a signal whose amplitude is a function of frequency and aspect angle. The signal received by the radar is a mix of the signals emitted by these sources. Consequently, this problem of separating objects can be studied using the sources classification methods.

Presently, our priority has been turned to the full exploitation of the wavelet coefficients, in order to improve the detection of a specific class of reflectors, and more globally in order to obtain a better discrimination of targets.

\section{References}

1 Carrara, W.G., Goodman, R.S., and Majewski, R.M.: 'Spotlight synthetic aperture radar' (Artech House, Boston, London, 1994)

2 Mensa, D.L.: 'High resolution radar imaging' (Artech House, USA, 1981)

3 Soumekh, M.: 'Fourier array imaging' (PTR Prentice Hall, Englewood Cliffs, 1994)

4 Soumekh, M.: 'Synthetic aperture radar signal processing' (John Wiley \& Sons, Inc., 1999)

5 Gough, P.T., and Hawkins, D.W.: 'Unified framework for modern synthetic aperture imaging algorithms', Int. J. Imaging Syst. Technol., 1997, 8, pp. 343-358

6 Potter, L.C., Chiang, D.M., Carrire, R., and Gerry, M.J.: 'A geometrical theory of diffraction (GTD)-based parametric model for radar scattering', IEEE Trans. Antennas Propag., 1995, 43, pp. $1058-1067$

7 Potter, L.C., and Moses, R.L.: 'Attributed scattering centers for SAR ATR', IEEE Trans. Image Process., 1997, 6, pp. 79-91

8 Van Der Merwe, A., Gerry, M.J., Potter, L.C., and Gupta, I.J.: 'Feature estimation performance using a two-dimensional parametric model of radar scattering', SPIE, 1997, 3070, pp. 322-333

9 Bertrand, J., Bertrand, P., and Ovarlez, J.P.: 'Dimensionalized wavelet transform with application to radar imaging'. Proc. IEEE-ICASSP, Toronto, Canada, May 1991
10 Bertrand, J., Bertrand, P., and Ovarlez, J.P.: 'Frequency directivity scanning in laboratory radar imaging', Int. J. Imaging Syst. Technol., 1994, 5, pp. 39-51

11 Ovarlez, J.P., Vignaud, L., Castelli, J.C., Tria, M., and Benidir, M.: 'Analysis of SAR images by multidimensional wavelet transform', IEE-Radar Sonar Navig., 2003, 150, (4), pp. 234-241 (special issue on time-frequency analysis for synthetic aperture radar and feature extraction)

12 Tria, M., Ovarlez, J.P., Vignaud, L., Castelli, J.C., and Benidir, M.: 'SAR imaging using multidimensional continuous wavelet transform'. Proc. Eusipco'04, Eur. Signal Process. Conf., Vienna-Austria, September 2004

13 Castelli, J.C., and Bobillot, G.: 'I4D: a new approach to RCS imaging analysis'. Proc. AMTA, Boston, USA, November 1997

14 Mallat, S.: 'Wavelet tour of signal processing' (Academic Press, 1998)

15 Boutry, J.M.: 'ONERA airborne SAR facilities'. 2nd Int. Airborne Remote Sensing Conf., San Francisco, USA, June 1996

16 Tria, M., Ovarlez, J.P., Vignaud, L., Castelli, J.C., and Benidir, M. 'SAR imaging using multidimensional continuous wavelet transform'. Proc. Radar'04, Int. Conf. on Radar Syst., Toulouse-France, October 2004

17 Tria, M.: 'Imagerie Radar a Synthèse d'Ouverture Par Analyse en Ondelettes Continues Multidimensionnelles', $\mathrm{PhD}$ thesis, University of Paris XI, 2005

18 Colin, E., Tria, M., Titin-Schnaider, C., Ovarlez, J.P., and Benidir, M.: 'SAR imaging using the multidimensional continuous wavelet transform and applications to polarimetry and interferometry', Int. J. Imaging Syst. Technol., 2005, 14, (5), pp. 206-212

19 Colin, E., and Tria, M.: 'SAR imaging using multidimensiona continuous wavelet transform and applications to polarimetry and interferometry'. Proc. Radar'04, Int. Conf. on Radar Syst., Toulouse-France, October 2004

20 Duquenoy, M., Ovarlez, J.P., Ferro-Famil, L., Vignaud, L., and Pottier, E.: 'Study of dispersive and anisotropic scatterers behavior in radar imaging using time-frequency analysis and polarimetric coherent decompositions'. Proc. EUSAR, Dresden, Germany, 16-18 May 2006

21 Tria, M., Ovarlez, J.P., Vignaud, L., Castelli, J.C., and Benidir, M. 'Full exploitation of wavelet coefficients in radar imaging for improving the detection of a class of sources in the context of real data'. Proc. Eusipco'06, Eur. Signal Process. Conf., Florence-Italy, September 2006 